\title{
One-Year Recording of Cardiac Arrhythmias in a Non-Infected Population with Cardiac Implantable Devices During the COVID-19 Pandemic
}

\author{
Yao-Ji Wang (D) \\ Qi-Qi Jin \\ Cheng Zheng \\ Jia-Xuan Lin \\ Yi-Fan Lin $\mathbb{D}$ \\ Que Xu \\ Jin $\mathrm{Li}$ \\ Jia-Feng Lin
}

Department of Cardiology, The Second Affiliated Hospital and Yuying Children's Hospital of Wenzhou Medical University, Wenzhou, Zhejiang, People's Republic of China
Correspondence: Jia-Feng Lin; Jin Li Email linjiafeng_wzmcfey@I63.com; wdy0I15@I26.com
Objective: Coronavirus disease 2019 (COVID-19) was associated with a higher risk of arrhythmia in infected patients. However, there are no reports about the effect of the ongoing pandemic on arrhythmias in the non-infected population. We measured the arrhythmia burden in a non-infected population with cardiac implantable devices.

Methods: The arrhythmia burden during the COVID-19 pandemic was compared to a 6-month interval in the pre-COVID-19 period. The COVID-19 pandemic was divided into high-risk (17 January 2020 to 16 March 2020) and low-risk periods (17 March 2020 to 17 July 2020) according to whether there were locally infected patients. Arrhythmia burdens were compared among the pre-COVID-19, high-risk, and low-risk periods.

Results: A total of 219 patients with 1859 episodes were included. We observed a larger proportion of patients with atrial fibrillation (AF) during the COVID-19 pandemic $(38.36 \%$ vs $26.03 \%, p=0.006$ ). There was not significantly more ventricular arrhythmia during the COVID period than the pre-COVID-19 period $(p>0.05)$. During the high-risk period, daily frequency of non-sustained ventricular tachycardia (NSVT) $(0.0172,0.0475$ vs 0.0109 , $0.0164, p<0.05)$, atrial tachycardia (AT) $(0.0345,0.0518$ vs $0.0164,0.0219 p<0.05)$ and $\mathrm{AF}(0.0345,0.0432$ vs $0.0164,0.0186, p<0.05)$ and daily duration of NSVT $(0.1982,0.2845$ vs $0.0538,0.1640 p<0.05)$ were higher and longer than those in the pre-COVID-19 period. Regression modeling showed that the impact of COVID-19 pandemic lead to an increased onset of AF (odds ratio 2.465; $\mathrm{p}<0.01$ ). Patients with paroxysmal AF who had undergone a previous radiofrequency ablation had a lower burden of AF (incidence $21.43 \%$ vs $55.00 \%$, $P=0.049$, daily frequency $0.0000,0.0027$ vs $0.0000,241.7978, P=0.020$ ) during the pandemic.

Conclusion: The COVID-19 pandemic contributed to a higher burden of arrhythmias in non-infected patients. Patients would experience a lower burden of AF following radiofrequency ablation treatment, and this effect persisted during the pandemic.

Keywords: COVID-19, arrhythmia, cardiac implantable device, non-infected population, radiofrequency ablation

\section{Introduction}

Coronavirus disease 2019 (COVID-19), caused by severe acute respiratory syndrome coronavirus 2 (SARS-CoV-2) was a global pandemic that infected more than 200 million patients worldwide as of mid-September 2021. COVID-19 was associated with myocardial injury and a higher risk of arrhythmias in infected patients. Early reports from China suggested an overall cardiac arrhythmia incidence of $17 \%$ in hospitalized COVID-19 patients. ${ }^{1}$ A higher arrhythmia rate (44\%) was observed 
in COVID-19 patients admitted to the intensive care unit. ${ }^{2}$ Another observational report from Italy described a concomitant increase in out-of-hospital cardiac arrests with the increased cumulative incidence of COVID-19. ${ }^{3}$

Evidence derived from various studies was inconsistent regarding the effect of COVID-19 on arrhythmias. In a large implantable cardiac defibrillator (ICD) population in the US, there was a $32 \%$ reduction in ventricular arrhythmia requiring ICD therapies following implementation of lockdown measures. ${ }^{4}$ Another report described an increase in ventricular arrhythmias in the 2 weeks before the lockdown order, and ventricular arrhythmia incidence decreased dramatically during the lockdown. ${ }^{5}$ However, a report from Italy declared that the complete nationwide lockdown did not affect the incidence of arrhythmias in an urban cohort of patients with ICDs. ${ }^{6}$ All these studies investigated the impact of COVID19 on the incidence of ventricular arrhythmias in ICD recipients followed by remote monitoring. Nevertheless, remote monitoring does not allow the researcher to define the proportion of patients infected with the COVID-19. All these studies weighed the arrhythmia burden in the entire population without distinguishing infected from non-infected patients. As COVID infection itself is a well-recognized trigger for cardiac arrhythmias, therefore, it remains clear whether the variation in arrhythmia burden is directly correlated with the change of the proportion of infected people rather than the comprehensive effect of pandemic itself; none of these studies reflected the actual arrhythmia burden in most non-infected patients. Therefore, we measured the arrhythmia burden in the non-infected population during the COVID-19 pandemic using cardiac implantable device interrogation.

We had the following aims: 1) to compare the incidences of several arrhythmias between the pre-COVID-19 period and the COVID-19 period; 2) to compare the arrhythmia burden, defined by the combination of daily frequency and daily duration between the pre-COVID-19 period and the COVID-19 period; and 3) to identify potential factors giving rise to the increase of cardiac arrhythmias during the COVID-19 pandemic. A regression model was constructed to evaluate the association between the arrhythmia and each potential risk factor.

\section{Methods}

\section{Study Design}

This was a cohort study conducted at the Second Affiliated Hospital and Yuying Children's Hospital of Wenzhou
Medical University, Wenzhou, China. The study period was selected based on the epidemiology of the COVID19 pandemic in the area. Wenzhou, China, was the first area to experience an outbreak of COVID-19 outside Wuhan, where the first case was diagnosed on 17 January 2020, and the final case was recorded on 16 March 2020. We selected 17 January 2020 to 17 July 2020 as the COVID-19 period and 17 July 2019 to 16 January 2020 as the pre-COVID-19 period. To measure the influence of the COVID-19 pandemic locally, the COVID-19 pandemic period was divided into a highrisk period (17 January 2020 to 16 March 2020, 58 days) and a low-risk period (17 March 2020 to 17 July 2020, 125 days) according to whether there were infected patients locally. The details are shown in Figure 1A. The devices detected the arrhythmias were collected during the COVID-19 period and compared to those occurring during the pre-COVID-19 period. Comparisons were also made among pre-COVID-19, high-risk, and low-risk periods.

\section{Study Population}

Inclusion criteria included no SARS-CoV-2 infection as determined by polymerase chain reaction testing of a nasopharyngeal sample and having an implantable device including permanent pacemakers, ICD, cardiac resynchronization therapy (CRT), or a cardiac resynchronization therapy defibrillator (CRT-D). Between August 2020 and May 2021, 1235 patients followed up at our Electrophysiology and Cardiac Pacing Unit. The exclusion criteria and arrhythmia diagnosis process are shown in Figure 1B. (a) new antiarrhythmic drugs or interventional treatments during the study period; (b) incomplete implantable device-detected episodes because of data clearance after outpatient follow-up (Medtronic) or data coverage by new arrhythmias (St. Jude, Boston Scientific, and BIOTRONIK); (c) implantation after 17 July 2019; (d) the implantable device could not record intracardiac electrograms (inECGs) of episodes; and (e) replacement of implantable device during the study period. The final cohort consisted of 219 patients. Demographic, medical history, and echocardiographic data were collected from electronic medical records. The medical history was defined by eligible diagnosis codes or prescription fills.

\section{Diagnostic Process}

The high-rate episode detection algorithm, used by all devices in this study, was triggered when the atrial/ventricular rate exceeded the programmed atrial/ventricular rate 
A

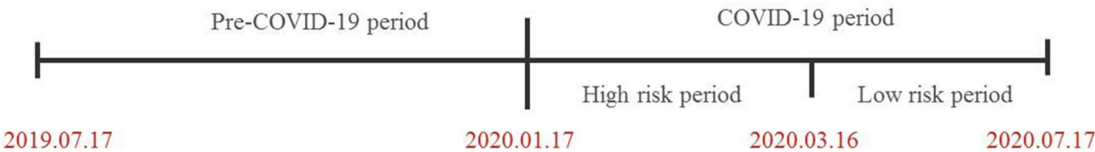

B

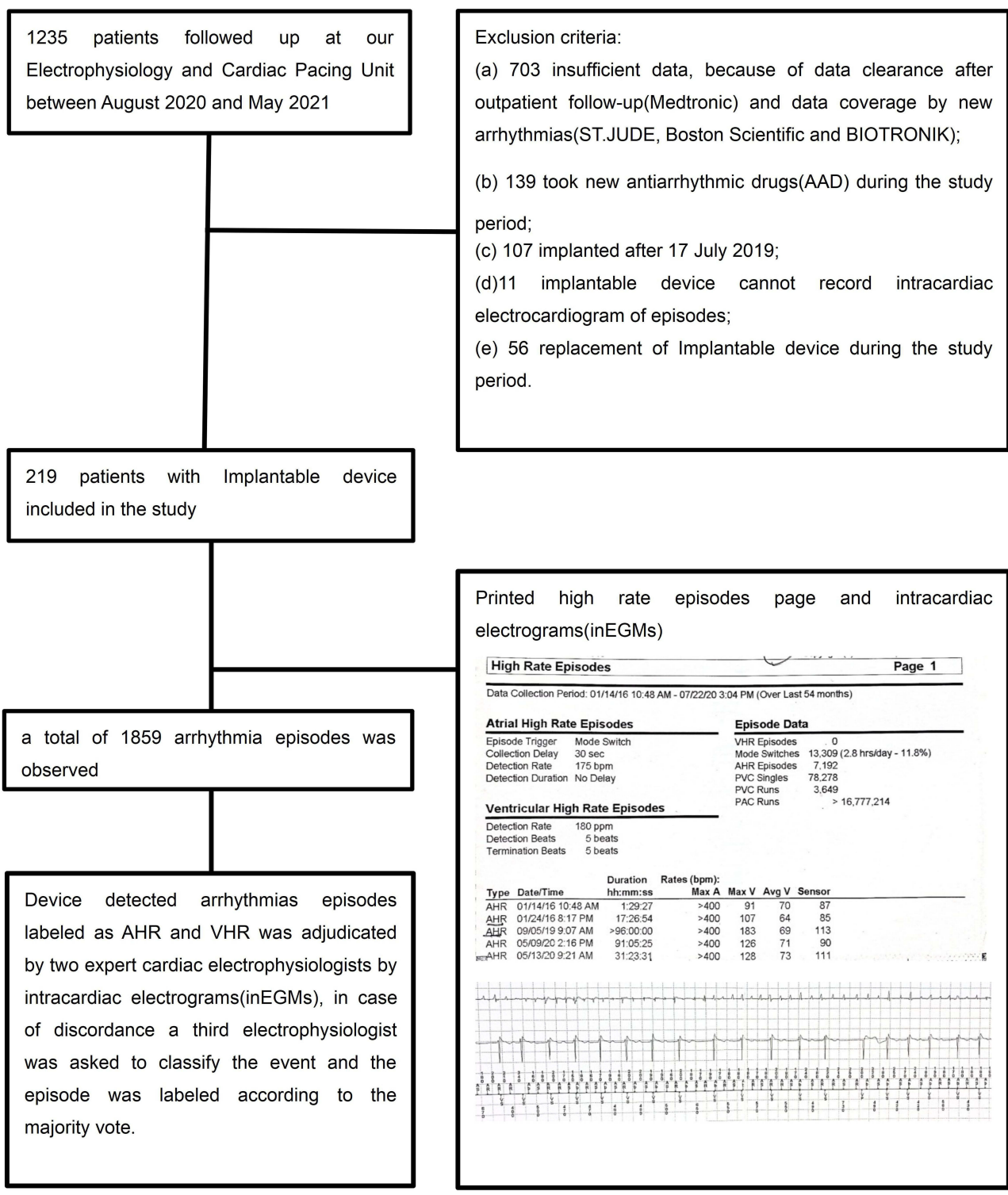

Figure I (A) Timeline illustrating crucial periods of the study. The first case was diagnosed on 17 January 2020, and the final one recovered on 16 March 2020 . We selected 17 January 2020 to 17 July 2020 as the COVID-19 period and 17 July 2019 to 16 January 2020 as the pre-COVID-19 period. To truly assess the influence of the COVID-19 pandemic locally, the COVID-19 pandemic period was divided into the high-risk period (17 January 2020 to 16 March 2020) and low-risk period (I7 Mar 2020 to 17 July 2020) according to whether the existence of locally infected patients. (B) The diagram shows the study population's selection and diagnosis process. The figure in diagram give an example of high rate episodes summary detected by Medtronic, which shows data collection period is 01/14/2016 10:48am-07/22/20 3:04pm (over last 54 months), atrial high rate episodes(AHR) was triggered when the atrial rate exceeded $175 \mathrm{bpm}$, the stored atrial inEGM is generated 30 seconds after the tachycardia meets AHR detection criteria. Ventricular high rate episodes(VHR) was triggered when the ventricular rate exceeded I80bpm and sustained for 5 beats. This figure depicts (top to bottom) 5 recorded episodes of AHR and provide the onset date and time along with the duration and stored intracardiac electrograms.

for high-rate episode detection and when the atrial/ventricular tachyarrhythmia was sustained for the minimum programmed number of complexes. The recording of the detected episode was terminated when the atrial/ ventricular rate decreased below the detection rate. We used the definition of the device's manufacturer to classify the arrhythmia episodes and print inECGs. ${ }^{7,8}$ To reduce the selection bias arising from different algorithms across 
devices, device-detected arrhythmia episodes labeled as atrial or ventricular high-rate episodes were adjudicated by two expert cardiac electrophysiologists using inEGMs. In case of disagreement, a third electrophysiologist was asked to classify the event, and the episode was labeled according to the majority vote. The characteristics of the various arrhythmia inEGMs are shown in Figure 2.

\section{Statistical Analysis}

Continuous variables were expressed as mean \pm standard deviation for data with normal distribution and median and inter-quartile range for data with skewed distributions. The Kolmogorov-Smirnov test was used to evaluate the normality of the distribution for continuous variables. The event rate in the pre-COVID-19 period was compared to the COVID-19 period or high-risk and low-risk period using the chi-squared test. Per-patient daily duration and daily frequency in the study period were calculated as the total occurrence times and duration divided by the number of days in the period (eg, during the high-risk period, 24 patients had NSVT, if someone had ten episodes of NSVT with a total duration of 40 seconds, and the high-risk period lasted 58 days; therefore the incidence of NSVT was $24 / 219$, the daily patient duration was $40 / 58$ seconds and daily frequency was $10 / 58$ times). The daily frequency and daily duration across periods were compared using the MannWhitney $U$-test (between pre-COVID-19 and COVID19 periods) and the Kruskal-Wallis $H$-test (for preCOVID-19, high-risk, and low-risk periods).

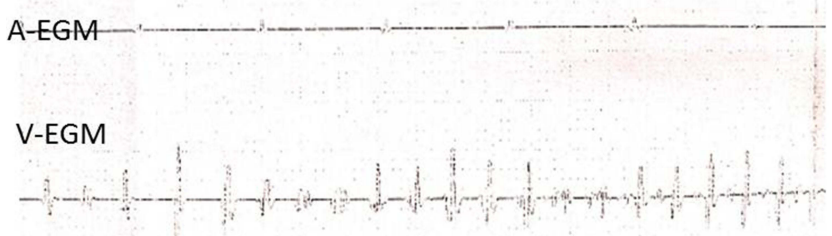

A

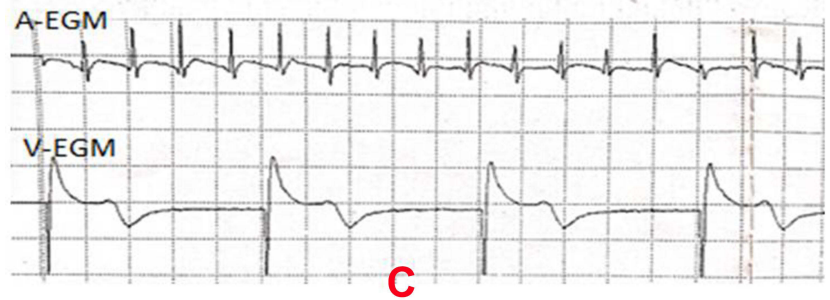

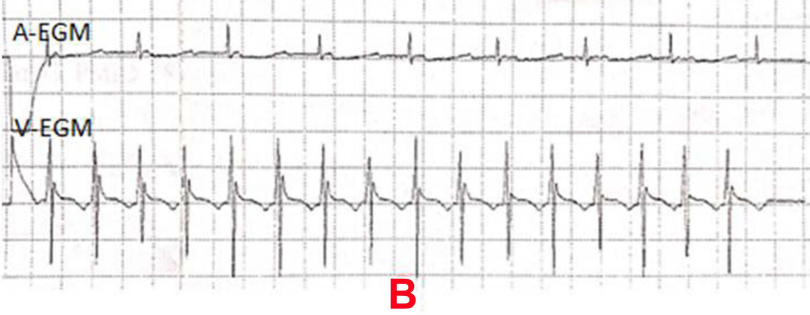

B

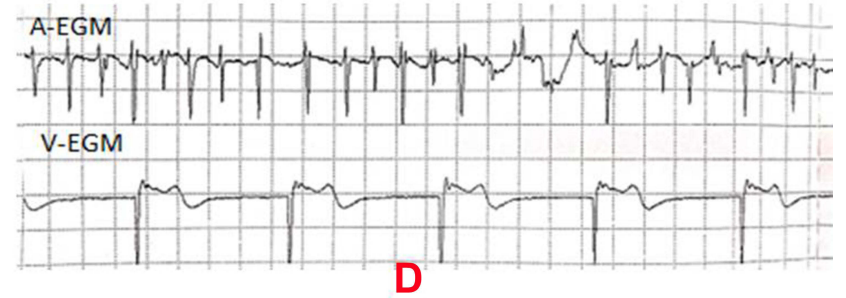

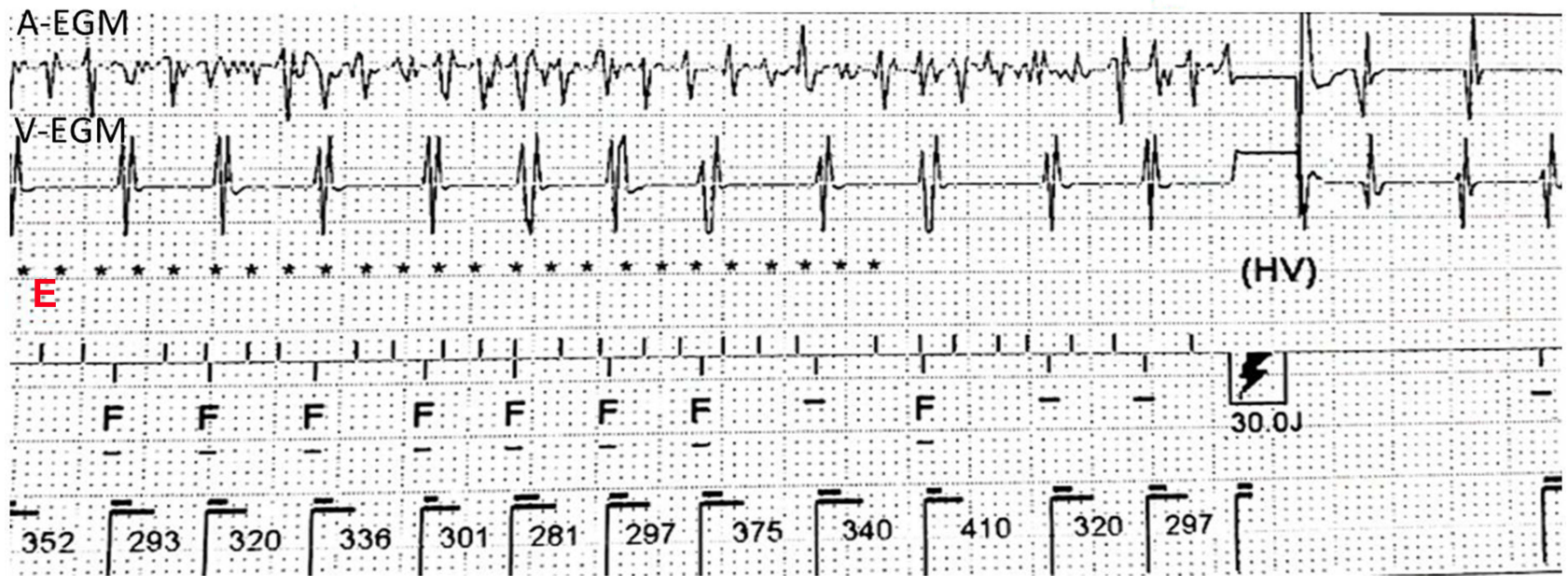

Figure 2 Intracardiac electrograms of arrhythmia. (A) Confirmed episode of SVT, A-A interval $=64 \mathrm{I}-1289 \mathrm{~ms}$, rate $=47-94 \mathrm{bpm}$; V-V interval = I72-273 ms, rate = 220$348 \mathrm{bpm}$; (B) Confirmed episode of NSVT, A-A interval = $590 \mathrm{~ms}$ (atrial pacing), rate $=47-94 \mathrm{bpm} ; \mathrm{V}-\mathrm{V}$ interval $=290-310 \mathrm{~ms}$, rate $=194-207 \mathrm{bpm}$, duration $=4920 \mathrm{~ms}$. (C) Confirmed episode of AT, regular A-A interval $=220 \mathrm{~ms}$, rate $=273 \mathrm{bpm}$; $\mathrm{V}-\mathrm{V}$ interval $=1000 \mathrm{~ms}$ (ventricular pacing), rate $=60 \mathrm{bpm}$; (D) confirmed episode of $A F$, A-A interval $=170-210 \mathrm{~ms}$, rate $=286-352 \mathrm{bpm}$; V-V interval $=980 \mathrm{~ms}$ (ventricular pacing), $6 \mathrm{I} \mathrm{bpm}$. (E) False-positive example of electrograms (inEGMs) stored as VT/VF. The episode A-A interval $=80-240 \mathrm{~ms}$, rate $=250-750 \mathrm{bpm} ; \mathrm{V}-\mathrm{V}$ interval $=28 \mathrm{I}-410 \mathrm{~ms}, 146-213 \mathrm{bpm}$, according to intracavitary electrogram, according to inEGM, we diagnosed as AF with fast ventricular rate. Implantable device diagnosed this episode as VT/VF and given a 30J shock.

Abbreviations: A-ECG, atrial intracardiac electrogram; V-ECG, ventricular intracardiac electrogram; ms, millisecond; bpm, beats per minute. 
A multivariate log-binomial regression model was established with adjustment for potential baseline confounders to evaluate the risk factors of arrhythmias further. We constructed a generalized estimation equation to evaluate the impacts of the COVID-19 pandemic on the risk of arrhythmias. Statistical analysis was performed using SPSS Statistics software, version 20.0 (IBM, Armonk, NY, USA). Two-sided p-values $<0.05$ indicated statistical significance.

\section{Follow-Up}

This was a retrospective study after cardiac implantable device implantation in patients regularly followed up at our Electrophysiology and Cardiac Pacing Unit. All data on these episodes were obtained from a record review. We collected arrhythmia episodes, downloaded the intracardiac electrocardiogram of each patient, and asked each patient about antiarrhythmic drugs, interventional therapy, other treatments, and health status during the study period in detail. We exclude the patient from the study once treatment or other factors could influence the arrhythmia burden. In addition, when the severe arrhythmia episodes requiring intervention were monitored at device interrogation. The results were disseminated to patients truthfully, and they would obtain necessary treatment, including antiarrhythmic drugs or radiofrequency ablation.

\section{Results}

\section{Patient Characteristics}

In this 366-day observational study, a total of 219 patients were included (age, $68.86 \pm 12.03$ years; male, 60.7\%). Etiologies leading to implantation were sick sinus syndrome in 85 (38.81\%), atrial-ventricular block in 53 $(24.20 \%)$, ejection fraction $<35 \%$ for primary prevention $46(21.00 \%)$, and idiopathic ventricular arrhythmias in 35 (15.99\%). A total of 138 (63.01\%) patients had permanent pacemakers, 7 (3.20\%) had undergone CRT, 40 (18.26\%) had ICDs, and 34 (15.53\%) had CRT defibrillators. The implantable cardiac device was from different manufacturers, 37 from BIOTRONIK, 31 from Boston Scientific, 54 from Medtronic, and 97 from St. Jude. Characteristics of the study population are provided in Table 1.

\section{Incidence of Various Types of Arrhythmias}

During the pre-COVID-19 period, 5 (2.28\%) patients had sustained ventricular tachycardia (SVT), 36 (16.44\%) had
Table I Baseline Characteristics of Study Population

\begin{tabular}{|c|c|}
\hline \multicolumn{2}{|l|}{ Characteristics } \\
\hline Demographic and medical history & \\
\hline Male, n (\%) & $133(60.7)$ \\
\hline Age (years), (mean $\pm S D)$ & $68.86 \pm 12.03$ \\
\hline BMI $(\mathrm{kg} / \mathrm{m} 2),($ mean $\pm \mathrm{SD})$ & $23.77 \pm 3.06$ \\
\hline Habitual alcohol intake, n (\%) & $4 \mid(\mid 8.72)$ \\
\hline Smoker, n (\%) & $51(23.3)$ \\
\hline Heart failure, $n(\%)$ & $70(32.0)$ \\
\hline Coronary heart disease, $\mathrm{n}(\%)$ & $65(29.7)$ \\
\hline Hypertension, n (\%) & $138(63.0)$ \\
\hline Diabetes, n (\%) & $58(26.48)$ \\
\hline DCM, n (\%) & $15(6.8)$ \\
\hline HCM, n (\%) & $7(3.2)$ \\
\hline Atrial fibrillation history, $\mathrm{n}(\%)$ & $58(26.48)$ \\
\hline Chronic kidney disease, n (\%) & $24(10.96)$ \\
\hline Peripheral artery disease, $\mathrm{n}(\%)$ & $129(28.90)$ \\
\hline COPD, n (\%) & $10(4.57)$ \\
\hline Hyperthyroidism, n (\%) & $9(4.11)$ \\
\hline Stroke history, n (\%) & $17(7.76)$ \\
\hline Echocardiographic parameters & \\
\hline $\begin{array}{l}\text { Left atrial diameter }(\mathrm{mm}) \text { [median, } \\
\text { range] }\end{array}$ & $40.53(28.76)$ \\
\hline LVEDD $(\mathrm{cm})$ [median, range] & $51.44(35.96)$ \\
\hline LVESD $(\mathrm{cm})$ [median, range] & $35.97(23.85)$ \\
\hline LV systolic dysfunction, n (\%) & \\
\hline None & $156(7 \mid .23)$ \\
\hline Mild & $19(8.68)$ \\
\hline Moderate & $20(9.13)$ \\
\hline Severe & $24(10.96)$ \\
\hline LVEF (\%)[median, range] & $57.53(17.75)$ \\
\hline Indication for implanted devices, $\mathrm{n}(\%)$ & \\
\hline Sick sinus syndrome (SSS) & $85(38.81)$ \\
\hline Atrial ventricular block (AVB) & $53(24.20)$ \\
\hline LVEF $<35 \%$ & $46(21.00)$ \\
\hline IVT & $35(15.99)$ \\
\hline Types of implanted devices, $n(\%)$ & \\
\hline Permanent pacemaker & $138(63.0 \mid)$ \\
\hline CRT & $7(3.20)$ \\
\hline$I C D$ & $40(18.26)$ \\
\hline CRTD & $34(15.53)$ \\
\hline Manufacturer, $\mathrm{n}(\%)$ & \\
\hline BIOTRONIK & $37(16.89)$ \\
\hline Boston Scientific & $31(14.16)$ \\
\hline Medtronic & $54(24.66)$ \\
\hline St-jude & $97(4 I .55)$ \\
\hline
\end{tabular}

Abbreviations: BMI, body mass index; DCM, dilated cardiomyopathy; HCM, hypertrophic cardiomyopathy; COPD, chronic obstructive pulmonary disease; LVESD, left ventricular end-systolic diameter; LVESD, left ventricular end-systolic diameter; LVEF, left ventricular ejection fraction; IVT, idiopathic ventricular tachycardia; ICD, implantable cardiac defibrillator; CRT, cardiac resynchronization therapy; CRT-D, cardiac resynchronization therapy defibrillator. 


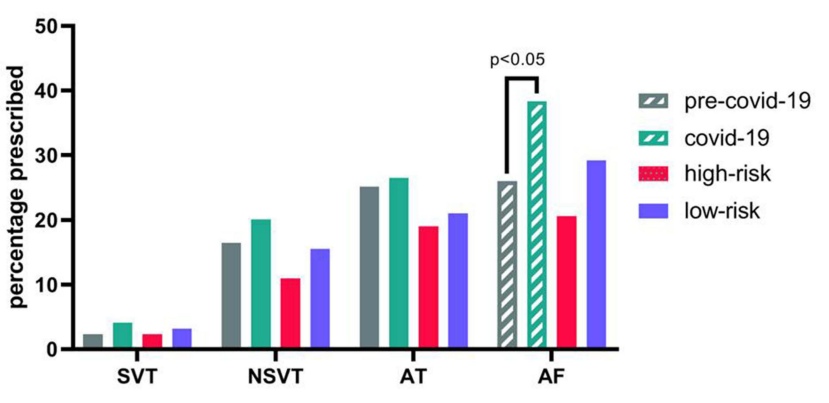

Figure 3 The incidence of arrhythmias across periods. Patients suffering from AF in COVID-19 pandemic more than patients in the pre-COVID-19 period $(P<0.05)$, No significant changes were observed for the incidence of SVT, VT, AT compared among different periods.

Abbreviations: AT, atrial tachycardia; AF, atrial fibrillation; SVT, sustained ventricular tachycardia; NSVT, non-sustained ventricular tachycardia.

Non-sustained ventricular tachycardia (NSVT), 55 (25.11\%) had Atrial tachycardia (AT), and 57 (26.03\%) had atrial fibrillation (AF). During the COVID-19 period, 9 (4.11\%) had SVT, 44 (20.09\%) had NSVT, 58 (26.48\%) had AT, and 84 (38.36\%) had AF. During the high-risk COVID-19 period, 5 (2.28\%) had SVT, 24 (10.96\%) had NSVT, 27 (12.33\%) had AT, and 45 (20.55\%) had AF. During the low-risk COVID-19 period, 7 (3.20\%) patients had SVT, 34 (15.53\%) had NSVT, 46 (21.00\%) had AT, and $64(29.22 \%)$ had AF.

There were $84(38.36 \%)$ patients who suffered from AF during the COVID-19 period, more than the 57 $(26.03 \%)$ who had AF in the pre-COVID-19 period $(p=0.006)$. There was no increase in ventricular arrhythmias and AT incidence during the COVID-19 period compared to the pre-COVID-19 period.

When the studied period was divided into pre-COVID -19 , high-risk, and low-risk periods, there was no significant difference in SVT, NSVT, AT, or AF incidence among these three periods (all $p>0.05$; Figure 3 ).

\section{Comparison of Arrhythmia Burden}

There was no evidence of increased daily frequency and duration of SVT, NSVT, AT, or AF when comparing the whole COVID-19 period to the pre-COVID-19 period. However, the daily NSVT frequency in the pre-COVID -19 period was less than it in the high-risk and low-risk periods $(p<0.05)$, and the daily NSVT duration in the high-risk period was significantly longer than during the pre-COVID-19 period (0.1982s Versus 0.0538, $p=0.023$ ). The daily AT frequency in the high-risk period was more significant than the pre-COVID-19 period ( 0.0345 versus 0.0164 times, $p=0.034$ ). The daily AF frequency was similar in the high-risk and low-risk periods, while it was relatively lower in the pre-COVID-19 period $(\mathrm{P}<0.05)$ (Table 2).

The per-patient daily arrhythmia frequency and duration among significantly different groups was illustrated to present the results more intuitively. We constructed a linear regression model for AT, AF, and NSVT in Figure 4A. During the COVID-19 period, the daily frequency of NSVT, AT, and AF increased month by month. In addition, we found that daily NSVT duration was correlated in parallel with the evolution of the COVID-19 pandemic. There was a sudden increase in daily NSVT duration after the first COVID-19 case in Wenzhou, China, was declared, and after the final case recovered on 16 March 2020, the per-patient daily NSVT duration dropped significantly (Figure 4B).

Table 2 Comparison of the Mean of Arrhythmia Burden Across Periods

\begin{tabular}{|c|c|c|c|c|c|c|c|c|c|}
\hline & & Pre-COVID-19 & COVID-19 & High-Risk & Low-Risk & $F_{1} / U_{1}$ & $P_{1}$ & $\mathbf{F}_{2} / \chi^{2}$ & $\mathbf{p}_{2}$ \\
\hline \multirow[t]{2}{*}{ SVT } & Frequency & $0.0186 \pm 0.0126$ & $0.0346 \pm 0.0252$ & $0.0759 \pm 0.0707$ & $0.0400 \pm 0.0330$ & 3.5 & 0.087 & 2.2 & 0.151 \\
\hline & Duration & $0.907,2.1530$ & I.2022, 3.3688 & $2.5862,10.8880$ & I.3920, 2.2960 & 18 & 0.606 & 1.9 & 0.380 \\
\hline \multirow[t]{2}{*}{ NSVT } & Frequency & $0.0109,0.0164^{b c}$ & $0.0164,0.0314$ & $0.0172,0.0475^{\mathrm{a}}$ & $0.0240,0.042^{\mathrm{a}}$ & 616 & 0.082 & 18 & 0.000 \\
\hline & Duration & $0.0538,0.1640^{b}$ & $0.0710,0.1462$ & $0.1982,0.2845^{a}$ & $0.6080,0.3680$ & 273 & 0.580 & 7.6 & 0.022 \\
\hline \multirow[t]{2}{*}{ AT } & Frequency & $0.0164,0.0219^{b}$ & $0.0219,0.045$ & $0.0345,0.0518^{\mathrm{a}}$ & $0.0280,0.0640$ & 1565 & 0.861 & 7.3 & 0.025 \\
\hline & Duration & $0.8049,3.586 \mathrm{I}$ & $0.6448,3.2869$ & $2.0345,8.1379$ & $0.7480,5.0100$ & 1183 & 0.901 & 1.9 & 0.378 \\
\hline \multirow[t]{2}{*}{$A F$} & Frequency & $0.0164,0.0186^{b c}$ & $0.0219,0.0383$ & $0.0345,0.0432^{\mathrm{a}}$ & $0.0280,0.0640^{\mathrm{a}}$ & 1967 & 0.070 & 19 & 0.000 \\
\hline & Duration & |I.1667, 503.63|I & $24.5792,429.2486$ & $40.74 \mid 4,631.0173$ & $34.2560,529.8260$ & 1125 & 0.248 & 2.2 & 0.329 \\
\hline
\end{tabular}

Notes: $F_{1} / U_{1}, p_{1}$ compare pre-COVID-19 period with COVID-19 period; $F_{2} / \chi^{2}, p_{2}$ compared with pre-COVID-19 period, high-risk period and Low-risk period. ${ }^{2} C o m p a r e d$ with the pre-COVID-19 group, and $p<0.05$; ${ }^{b}$ Compared with the high-risk group, and $p<0.05$; ${ }^{\circ}$ Compared with the low-risk group, and $p<0.05$.

Abbreviations: SVT, sustained ventricular tachycardia; NSVT, nonsustained ventricular tachycardia; AT, atrial tachycardia; AF, atrial fibrillation. 

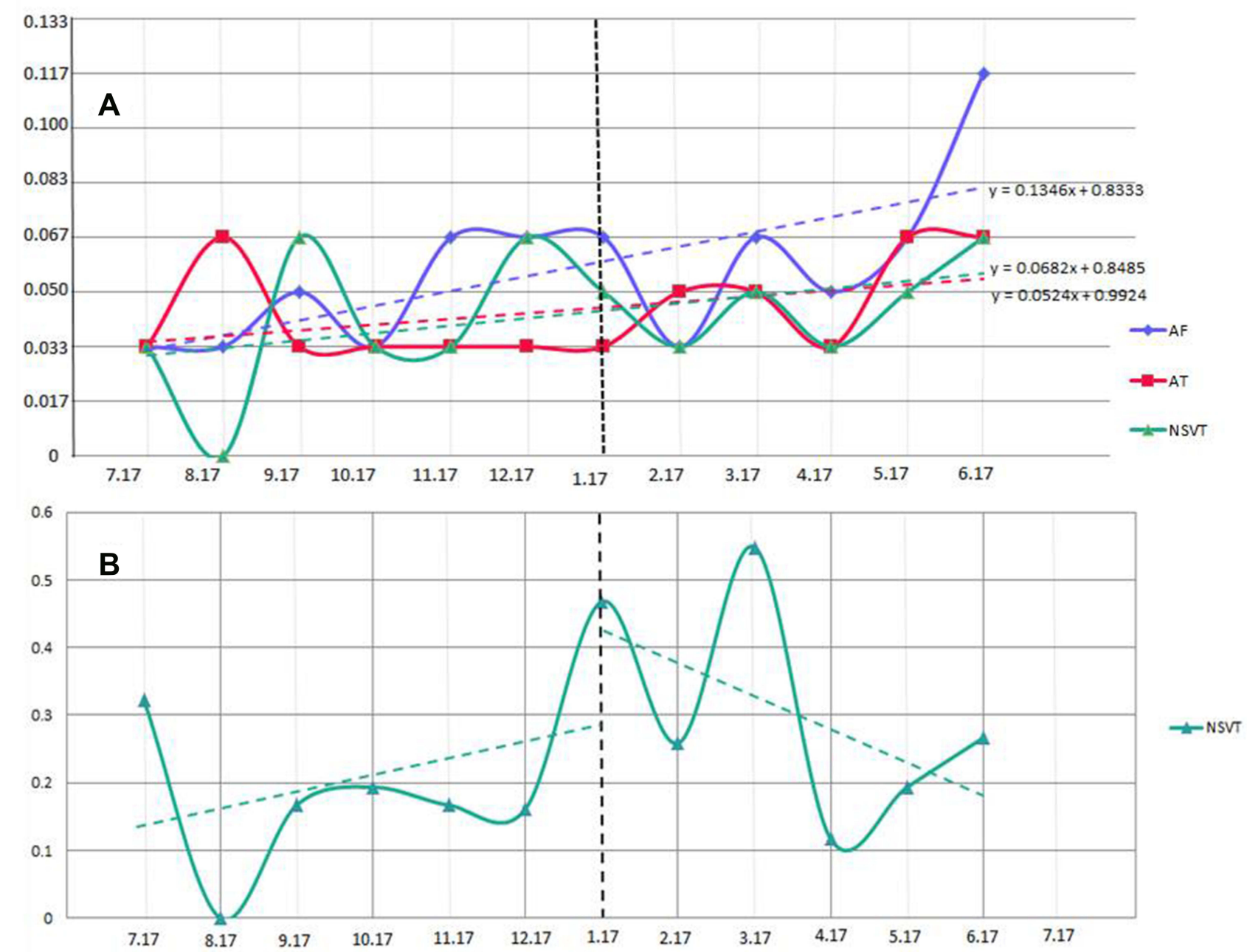

Figure 4 Evolution of the burden of cardiac arrhythmias. (A) The mean of per-patient daily NSVT, AT, and AF occurrences per month. (B) The mean of per-patient NSVT duration per month. The dotted line: Ist COVID-19 case in Wenzhou.

Abbreviations: AT, atrial tachycardia; AF, atrial fibrillation; NSVT, non-sustained ventricular tachycardia.

\section{Potential Risk Factors for Arrhythmia}

In our log-binomial regression and generalized estimation models, baseline variables that were considered clinically relevant and candidate variables with $p$-values $<0.2$ on univariate analysis were included in the multivariable model to ensure parsimony of the final model. ${ }^{9}$ After adjusting for all confounding factors, we used a generalized estimation equation to evaluate the impact of the COVID-19 pandemic on the risk of any arrhythmias. We found the impact of the COVID-19 pandemic was a significant risk factor for occurrence $\mathrm{AF}$ in the non-infected population (odds ratio 2.465, 95\% confidence interval, 1.324-4.553, $p<0.01)$. The COVID-19 pandemic had no impact on SVT, NSVT, or AT in our sample. The other potential risk factors for arrhythmia are shown in Figure 5.

\section{Previous RFCA Contributed to} a Reduction of AF Burden During the COVID Period

AF incidence during the COVID-19 pandemic was higher, and the impact of pandemic was an independent risk factor to promote the onset of AF in non-infected patients. Therefore, during the pandemic, we primarily focused on identifying any method to reduce the AF burden. Interventional therapy might be an effective, long-lasting solution for these recurrent malignant arrhythmias and should be considered. Therefore, we retrospectively evaluated 34 patients with paroxysmal AF; 14 underwent previous RFCA to control heart rhythm, while the others still suffered from paroxysmal AF. We compared the incidence, per-patient daily duration, and daily frequency of the two groups across periods. 


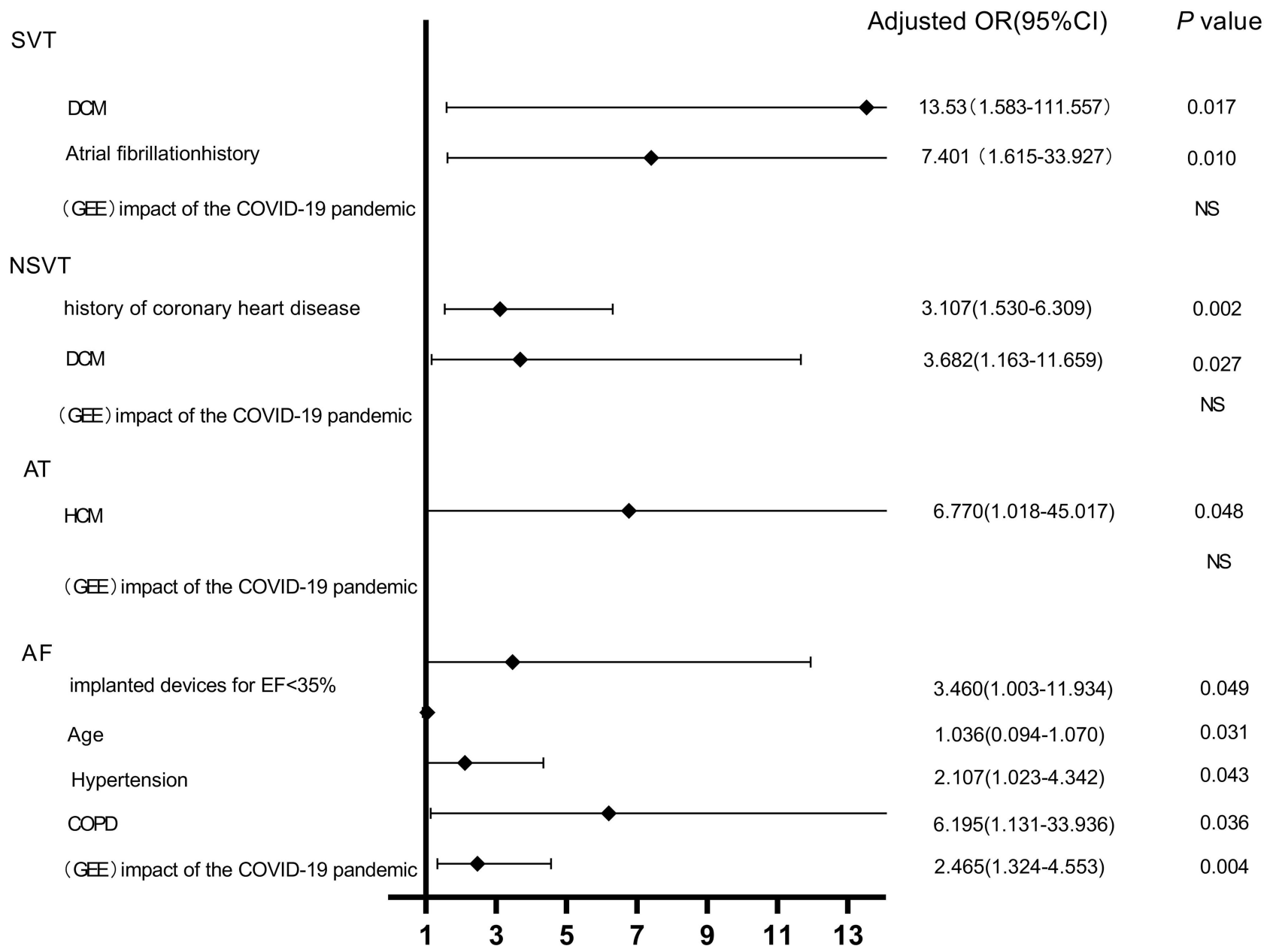

Figure 5 Logistic regression and generalized estimation equation analysis predicting the risk of arrhythmia during the COVID-19 epidemic.

Abbreviations: OR, odds ratio; DCM, dilated cardiomyopathy; HCM, hypertrophic cardiomyopathy; COPD, chronic obstructive pulmonary disease; GEE, generalized estimation equation.

There were no significant differences in factors influencing the occurrence of $\mathrm{AF}$, including age, the proportion of implanted devices for LVEF $<35 \%$, hypertension, and chronic obstructive pulmonary disease (COPD) (Table 3). The incidence of AF in the COVID-19 period in the RFCA group was significantly lower than the non-RFCA group (21.43\% versus $\left.55.00 \% \chi^{2}=3.832, P=0.049\right)$, So as the daily AF frequency $(0.0000,0.0027$ versus 0.0000 , 241.7978, $P=0.020$ ); However, daily AF duration in RFCA group was similar with non-RFCA group $(P>$ 0.05). As shown above, patients with AF could experience lower incidence and daily AF frequency following RFCA to control heart rhythm. This effect persisted during the pandemic; therefore, we recommend RFCA for patients with paroxysmal AF to reduce the arrhythmia burden in another unpredictable pandemic (Table 3).

\section{Discussion}

In our analysis of 219 out-of-hospital non-infected patients over 12 months, there were 1859 arrhythmia episodes recorded. The main results of this study are as follows: (a) there was a more significant proportion of patients with $\mathrm{AF}$ during the COVID-19 pandemic, and no increase was observed VAs; (b) the daily NSVT, AT, AF daily frequency, and NSVT duration were higher during the high-risk pandemic than the pre-COVID-19 period; (c) the COVID-19 pandemic appeared to promote the onset of AF in a non-infected population; However, we find that arrhythmias are the consequence of systemic illness, such as hypertension, dilated cardiomyopathy (DCM), hypertrophic cardiomyopathy (HCM), COPD, and a history of coronary heart disease; (d) controlling heart rhythm by RFCA may help reduce the incidence and daily AF frequency, this effect persisted during COVID-19 pandemic. 
Table 3 Characteristics of Patients with Paroxysmal Atrial Fibrillation

\begin{tabular}{|c|c|c|c|c|}
\hline Characteristics & RFCA $(n=14)$ & Non-RFCA $(n=20)$ & $\chi^{2} / U$ & $p$ \\
\hline Age(years) & $68.86 \pm 9.28$ & $71.40 \pm 12.46$ & 0.01 & 0.995 \\
\hline Implanted devices for LVEF < 35\%, n (\%) & $3.00(21.43)$ & $3.00(15.00)$ & 0.234 & 0.672 \\
\hline Hypertension, n (\%) & $12.00(85.7 \mathrm{I})$ & $12.00(60.00)$ & 2.632 & 0.141 \\
\hline COPD, n (\%) & $0.00(0.00)$ & $1.00(5.00)$ & 0.721 & 1.000 \\
\hline \multicolumn{5}{|l|}{ Incidence, n (\%) } \\
\hline Pre-COVID-19 period & $0.00(0.00)$ & $7.00(35.00)$ & 6.170 & 0.026 \\
\hline COVID-19 period & $3.00(21.43)$ & $11.00(55.00)$ & 3.832 & 0.049 \\
\hline \multicolumn{5}{|l|}{ Frequency } \\
\hline Pre-COVID-19 period & 0.0000 & $0.0000,8.4597$ & 98 & 0.048 \\
\hline COVID-19 period & $0.0000,0.0027$ & $0.0000,241.7978$ & 80.5 & 0.020 \\
\hline High-risk period & $0.0000,0.0345$ & $0.0000,8.8966$ & 122.5 & 0.386 \\
\hline Low-risk period & $0.0000,0.0160$ & $0.0000,80.7320$ & 96 & 0.056 \\
\hline \multicolumn{5}{|l|}{ Duration } \\
\hline Pre-COVID-19 period & 0.0000 & $0.0000,0.0157$ & 98 & 0.027 \\
\hline COVID-I9 period & $0.0000,50.0068$ & $0.0191,0.0669$ & 107.5 & 0.183 \\
\hline High-risk period & $0.0000,325.8276$ & $0.0000,0.0129$ & 134 & 0.752 \\
\hline Low-risk period & $0.0000,141.6560$ & $0.0000,0.0440$ & 112 & 0.207 \\
\hline
\end{tabular}

Abbreviations: COPD, chronic obstructive pulmonary disease; RFCA, radiofrequency catheter ablation; LVEF, left ventricular ejection fraction.

Environmental stress (such as earthquakes, war, or sporting events) may increase the risk of cardiovascular events. $^{10,11}$ A growing body of evidence suggests that seasonal influenza pandemics trigger acute arrhythmias and coronary syndromes. Several studies reported that, during high activity influenza periods, patients were more likely to have ventricular arrhythmias treated with shock or anti-tachycardia pacing. ${ }^{12-14}$ Moreover, areas severely affected by the pandemic reported increased incidence of out-of-hospital cardiac arrest. ${ }^{3,15,16}$

In our study, the comprehensive effect of the COVID19 pandemic on society was objectively explored by investigating the arrhythmias burden in majority non-infected patients opposing the minority infected patients. We did not find any increased incidence of ventricular arrhythmias during the COVID-19 pandemic in non-infected patients, consistent with previous studies on the topic. ${ }^{4-6,17}$ However, we found that, during the COVID-19 pandemic, the incidence of AF was higher than during the preCOVID-19 period. Among the previous four studies on this topic, only one study from France mentioned atrial arrhythmia (the others only investigated ventricular arrhythmia burden); the authors demonstrated no changes regarding the evolution of atrial fibrillation/tachycardia episodes during the COVID-19 pandemic. ${ }^{17}$ The most likely explanation for the observed discrepancy was that the authors only studied the evolution of the incidence of atrial fibrillation/tachycardia episodes during the epidemic period and did not compare it with the pre-COVID-19 period. Additionally, we found a sudden increase in daily NSVT duration after the first COVID-19 case in Wenzhou, China, was declared and a significant drop after the final case recovered on 16 March 2020. We speculated that the COVID-19 pandemic could have triggered an integrated network of social factors, increasing the daily NSVT duration in the non-infected population. Similar results were shown in a study from France, where there was a sudden increase in the incidence of ventricular arrhythmia during the 2 weeks before the lockdown order. The two weeks before the lockdown order were associated explicitly with stress-inducing media coverage of the pandemic in France, including the first COVID-19 death in the country, the first televised presidential allocution on the topic, followed by a high number of COVID-19 cases and deaths.

One may hypothesize that this variation in AF incidence could be related to the stress generated by the outbreak. A study from Italy even revealed signs of psychological suffering after a protracted lockdown period. ${ }^{18}$ Other studies showed that, as the severity of the 
pandemic increased, the level of anxiety among the population also increased, bringing physical, mental, and psychological harm to people worldwide. ${ }^{19-22}$ These events contributed to the increase of adrenergic and dysfunction of the autonomic nervous system. Adrenergic inputs represent major neural triggers for atrial arrhythmias, ${ }^{23,24}$ especially in individuals with predisposing myocardial risk factors. Coumel et al reported that exercise and emotional stress could trigger adrenergic-mediated episodes of $\mathrm{AF}^{25}$ Another study reported inpatient cardiac monitoring during the pandemic and found that of new arrhythmias detected, $\mathrm{AF}$ was the most common. ${ }^{26}$

RFCA could help to reduce the burden of AF, and this effect persisted during the pandemic. Patients with paroxysmal AF was found owning a reduction of incidence and daily AF frequency by previous RFCA. Therefore, we recommend RFCA for patients with paroxysmal AF in cases of frequent arrhythmia recurrence in another unpredictable pandemic. Especially in another national lockdowns where individuals are asked to remain isolated in their homes to stop the spread of the disease, electrophysiologists are redeployed to perform emergency work outside electrophysiology, elective work was canceled, and cases were performed only in emergencies, ${ }^{27}$ and protect patients from the severe complication of AF, such as embolism and stroke.

A recent study reported that all areas of cardiology service sustained significant reductions in general hospitals. ${ }^{28}$ It seems to contradict the result we observed that some arrhythmia burdens were increased during the high-risk period. Fersia et al demonstrated that urgent service did not decrease during COVID-19; the authors found that, despite a significant reduction in elective coronary angiography, there was no change in the number of urgent coronary angiography or primary percutaneous coronary interventions performed. ${ }^{28}$ We believe the reduction of cardiology service provision did not indicate a decrease in cardiovascular events. The reasons for these reductions are multifactorial. First, fear and trepidation regarding exposure to COVID-19 in clinical settings may compel many to avoid calling for help. Patients might have been reluctant to seek medical help during the pandemic apart from life-threatening events. The second reason is the restructuring and prioritization of health services, prioritizing urgent cases, and reducing access to primary care. This phenomenon also concerned us, as we observed that some arrhythmia burden increased during the pandemic. We were apprehensive that arrhythmias would not be treated sufficiently, and some arrhythmia-induced disability is unavoidable. We encourage exploring more flexible and available methods from medical services to help diagnose arrhythmias and prescribe appropriate treatment during pandemics for most non-infected patients.

Finally, we evaluated the association between arrhythmia and potential risk factors. As shown in Figure 4, arrhythmias are likely the consequence of systemic illness, and the impact of the pandemic was not related to the onset of lifethreatening rhythms (SVT and NSVT), which may help reduce concerns experienced in the non-infected population during the pandemic. Furthermore, we could not ignore the non-infected people who had coronary heart disease, DCM, HCM, hypertension, COPD, or ejection fraction $<35 \%$ during the pandemic. These patients deserve special attention to ensure the early identification and intervention of arrhythmia.

\section{Limitations}

There were some limitations to the present study. First, this was a retrospective analysis, and several parameters could not be controlled for. We cannot claim with $100 \%$ certainty that the database contains accurately detected rhythms; despite device-detected arrhythmias, episodes were adjudicated by cardiac electrophysiologists to reduce potential bias. Second, this was a single-center study. We expect that sizable multi-center cohort studies will be conducted in the future to explore the long-term impact and the mechanism of the COVID-19 pandemic among the out-of-hospital non-infected population. Finally, in our sample, we did not systematically assess individual behavioral changes around physical activity, eating habits, and working from home arrangements; therefore, we could do no more than speculate regarding the association between the impact of the pandemic and arrhythmic events.

\section{Conclusion}

Based on our implantable device interrogation, we conclude that the COVID-19 pandemic contributed to a higher burden of arrhythmias in non-infected patients in a realworld setting. Patients with AF experienced a lower burden following RFCA to control heart rhythm, and this effect persisted during the pandemic; we recommend RFCA for patients with paroxysmal AF in cases of frequent arrhythmia recurrence in another unpredictable pandemic. We could not ignore the non-infected individuals who had coronary heart disease, DCM, HCM, hypertension, COPD, or ejection fraction $<35 \%$ during the 
pandemic. These patients deserve special attention to ensure the early identification and intervention of arrhythmia.

\section{Abbreviations}

COVID-19, coronavirus disease 2019; AT, atrial tachycardia; AF, atrial fibrillation; SVT, sustained ventricular tachycardia; NSVT, non-sustained ventricular tachycardia; RFCA, radiofrequency ablation; inECG, intracardiac electrogram; BMI, body mass index; DCM, dilated cardiomyopathy; HCM, hypertrophic cardiomyopathy; COPD, chronic obstructive pulmonary disease; LVESD, left ventricular end-systolic diameter; LVESD, left ventricular end-systolic diameter; LVEF, left ventricular ejection fraction; IVT, idiopathic ventricular tachycardia; ICD, implantable cardiac defibrillator; CRT, cardiac resynchronization therapy; CRT-D, cardiac resynchronization therapy defibrillator.

\section{Data Sharing Statement}

Data are available on reasonable request to the corresponding author. (Email: linjiafeng_wzmcfey@163.com).

\section{Ethics Approval and Informed Consent}

This study was approved by the Institutional Review Board at The Second Affiliated Hospital and Yuying Children's Hospital of Wenzhou Medical University (2021-K-36-01). Patient consent was waived as study a retrospective review of the medical files and anonymity was maintained all the way during data collection and manuscript writing. This study was conducted in accordance with the declaration of Helsinki.

\section{Author Contributions}

All authors contributed to data analysis, drafting or revising the article, have agreed on the journal to which the article will be submitted, gave final approval of the version to be published, and agree to be accountable for all aspects of the work.

\section{Funding}

This study was supported by Wenzhou Municipal Science and Technology Commission grant ZY2020018; and Department of Education of Zhejiang Province grant Y202044409. The funders had no role in study design, data collection and analysis, decision to publish, or preparation of the manuscript. The authors have reported that they have no relationships relevant to the contents of this paper to disclose.

\section{Disclosure}

The authors declare that they have no known competing financial interests or personal relationships that could have appeared to influence the work reported in this paper.

\section{References}

1. Wang $\mathrm{D}, \mathrm{Hu} \mathrm{B}, \mathrm{Hu} \mathrm{C}$, et al. Clinical characteristics of 138 hospitalized patients with 2019 novel coronavirus-infected pneumonia in Wuhan, China. JAMA. 2020;323(11):1061-1069. Erratum in: JAMA. 2021 Mar 16;325(11):1113. PMID: 32031570; PMCID: PMC7042881. doi:10.1001/jama.2020.1585

2. Guo T, Fan Y, Chen M, et al. Cardiovascular implications of fatal outcomes of patients with Coronavirus Disease 2019 (COVID-19). JAMA Cardiol. 2020;5(7):811-818. Erratum in: JAMA Cardiol. 2020 Jul 1;5(7):848. PMID: 32219356; PMCID: PMC7101506. doi:10.1001/jamacardio.2020.1017

3. Baldi E, Sechi GM, Mare C, et al. Out-of-hospital cardiac arrest during the covid-19 outbreak in Italy. $N$ Engl J Med. 2020;383 (5):496-498. PMID: 32348640; PMCID: PMC7204428. doi:10.1056/NEJMc2010418

4. O'Shea CJ, Thomas G, Middeldorp ME, et al. Ventricular arrhythmia burden during the coronavirus disease 2019 (COVID-19) pandemic. Eur Heart J. 2021;42(5):520-528. PMID: 33321517; PMCID: PMC7953962. doi:10.1093/eurheartj/ehaa893

5. Sassone B, Virzì S, Bertini M, et al. Impact of the COVID-19 lockdown on the arrhythmic burden of patients with implantable cardioverter-defibrillators. Pacing Clin Electrophysiol. 2021;44 (6):1033-1038. PMID: 34022067; PMCID: PMC8207039. doi: $10.1111 /$ pace. 14280

6. Malanchini G, Ferrari P, Leidi C, et al. Ventricular arrhythmias among patients with implantable cardioverter-defibrillator during the COVID-19 pandemic. $J$ Arrhythm. 2021;37(2):407-413. PMID: 33821178; PMCID: PMC8014654. doi:10.1002/joa3.12518

7. Nowak B, Sperzel J, Rauscha F, et al. Diagnostic value of onset-recordings and marker annotations in dual chamber pacemaker stored electrograms. Europace. 2003;5(1):103-109. PMID: 12504649. doi:10.1053/eupc.2002.0276

8. Pollak WM, Simmons JD, Interian A Jr, et al. Clinical utility of intraatrial pacemaker stored electrograms to diagnose atrial fibrillation and flutter. Pacing Clin Electrophysiol. 2001;24(4 Pt 1):424-429. PMID: 11341078. doi:10.1046/j.1460-9592.2001.00424.x

9. Kang SJ, Cho YR, Park GM, et al. Predictors for functionally significant in-stent restenosis: an integrated analysis using coronary angiography, IVUS, and myocardial perfusion imaging. JACC Cardiovasc Imaging. 2013;6(11):1183-1190. PMID: 24229771. doi:10.1016/j.jcmg.2013.09.006

10. Sato M, Fujita S, Saito A, et al. Increased incidence of transient left ventricular apical ballooning (so called 'Takotsubo' cardiomyopathy) after the mid-Niigata Prefecture earthquake. Circ J. 2006;70:947e953. doi:10.1253/circj.70.947

11. Serra Grima R, Carreno MJ, Tomas Abadal L, Brossa V, Ligero C, Pons J. Acute coronary events among spectators in a soccer stadium. Rev Esp Cardiol. 2005;58:587-591.

12. Madjid M, Connolly AT, Nabutovsky Y, et al. Effect of high influenza activity on risk of ventricular arrhythmias requiring therapy in patients with implantable cardiac defibrillators and cardiac resynchronization therapy defibrillators. Am J Cardiol. 2019;124(1):44-50. PMID: 31047651. doi:10.1016/j.amjcard.2019.04.011 
13. Russo V, Solimene F, Zanotto G, et al. Seasonal trend of ventricular arrhythmias in a nationwide remote monitoring database of implantable defibrillators and cardiac resynchronization devices. Int J Cardiol. 2019;275:104-106. PMID: 30327133. doi:10.1016/j. ijcard.2018.10.025

14. Mamas MA, Fraser D, Neyses L. Cardiovascular manifestations associated with influenza virus infection. Int J Cardiol. 2008;130 (3):304-309. PMID: 18625525. doi:10.1016/j.ijcard.2008.04.044

15. Craen A, Logan G, Ganti L. Novel coronavirus disease 2019 and subarachnoid hemorrhage: a case report. Cureus. 2020;12(4):e7846. PMID: 32483497; PMCID: PMC7253081. doi:10.7759/cureus.7846

16. Chan PS, Girotra S, Tang Y, et al. Outcomes for out-of-hospital cardiac arrest in the United States during the coronavirus disease 2019 pandemic. JAMA Cardiol. 2021;6(3):296-303. PMID: 33188678; PMCID: PMC7666759. doi:10.1001/jamacar dio. 2020.6210

17. Galand V, Hwang E, Gandjbakhch E, et al. Impact of COVID-19 on the incidence of cardiac arrhythmias in implantable cardioverter defibrillator recipients followed by remote monitoring. Arch Cardiovasc Dis. 2021;114(5):407-414. PMID: 34088625; PMCID: PMC8141722. doi:10.1016/j.acvd.2021.02.005

18. Orlandi M, Rosselli M, Pellegrino A, et al. Gender differences in the impact on physical activity and lifestyle in Italy during the lockdown, due to the pandemic. Nutr Metab Cardiovasc Dis. 2021:S0939-4753(21)00133-2. PMID: 33975735. doi:10.1016/j.numecd.2021.03.011

19. Wu T, Jia X, Shi H, et al. Prevalence of mental health problems during the COVID-19 pandemic: a systematic review and meta-analysis. J Affect Disord. 2021;281:91-98. PMID: 33310451; PMCID: PMC7710473. doi:10.1016/j.jad.2020.11.117

20. Wang S, Wen X, Dong Y, et al. Psychological influence of Coronavirus Disease 2019 (COVID-19) pandemic on the general public, medical workers, and patients with mental disorders and its countermeasures. Psychosomatics. 2020;61(6):616-624. PMID: 32739051; PMCID: PMC7255244. doi:10.1016/j.psym.2020.05.005
21. Coelho CM, Suttiwan P, Arato N, et al. On the nature of fear and anxiety triggered by COVID-19. Front Psychol. 2020;11:581314. PMID: 33240172; PMCID: PMC7680724. doi:10.3389/fpsyg.2 020.581314

22. Qiu J, Shen B, Zhao M, et al. A nationwide survey of psychological distress among Chinese people in the COVID-19 epidemic: implications and policy recommendations. Gen Psychiatry. 2020;33(2): e100213. Erratum in: Gen Psychiatr. 2020 Apr 27;33(2): e100213corr1. PMID: 32215365; PMCID: PMC7061893. doi:10.1136/gpsych-2020-100213

23. Tan AY, Verrier RL. The role of the autonomic nervous system in cardiac arrhythmias. Handb Clin Neurol. 2013;117:135-145. PMID: 24095122. doi:10.1016/B978-0-444-53491-0.00012-2

24. Franciosi S, Perry FKG, Roston TM, et al. The role of the autonomic nervous system in arrhythmias and sudden cardiac death. Auton Neurosci. 2017;205:1-11. PMID: 28392310. doi:10.1016/j.autneu.2 017.03 .005

25. Coumel P. Autonomic influences in atrial tachyarrhythmias. $J$ Cardiovasc Electrophysiol. 1996;7(10):999-1007. PMID: 8894942. doi:10.1111/j.1540-8167.1996.tb00474.x

26. Braunstein ED, Reynbakh O, Krumerman A, et al. Inpatient cardiac monitoring using a patch-based mobile cardiac telemetry system during the COVID-19 pandemic. J Cardiovasc Electrophysiol. 2020;31(11):2803-2811. PMID: 32852868; PMCID: PMC7461402. doi:10.1111/jce. 14727

27. Li J, Mazzone P, Leung LWM, et al. Electrophysiology in the time of coronavirus: coping with the great wave. Europace. 2020;22 (12):1841-1847. PMID: 32995866; PMCID: PMC7543596. doi:10.1093/europace/euaa185

28. Fersia O, Bryant S, Nicholson R, et al. The impact of the COVID-19 pandemic on cardiology services. Open Heart. 2020;7(2):e001359. PMID: 32855212; PMCID: PMC7454176. doi:10.1136/openhrt2020-001359
International Journal of General Medicine

\section{Publish your work in this journal}

The International Journal of General Medicine is an international, peer-reviewed open-access journal that focuses on general and internal medicine, pathogenesis, epidemiology, diagnosis, monitoring and treatment protocols. The journal is characterized by the rapid reporting of reviews, original research and clinical studies

\section{Dovepress}

across all disease areas. The manuscript management system is completely online and includes a very quick and fair peer-review system, which is all easy to use. Visit http://www.dovepress.com/ testimonials.php to read real quotes from published authors. 